ORIGINAL

\title{
Consumo de drogas y prácticas sexuales de los adolescentes de la ciudad de Medellin (Colombia)
}

\section{Drug use and sexual practices of teenagers in the city of Medellin (Colombia)}

\author{
Guillermo A. Castaño Pérez ${ }^{*}$; Eduardo Arango To- \\ BON*; Santiago Morales Mesa*; AleXander Rodrí- \\ guez Bustamante*; Carolina Montoya Montoya*
}

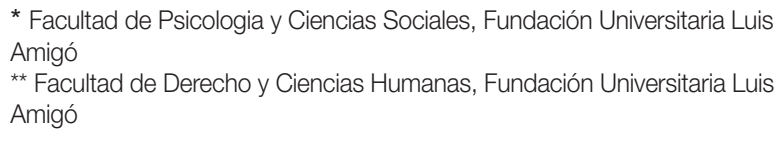

* Facultad de Psicologia y Ciencias Sociales, Fundación Universitaria Luis Amigó

** Facultad de Derecho y Ciencias Humanas, Fundación Universitaria Luis Amigó

\author{
Enviar correspondencia a: \\ Guillermo Castaño. \\ Facultad de Psicología y Ciencias Sociales. \\ Fundación Universitaria Luis Amigó. \\ Transversal 51 A. No. 67B - 90. Medellín (Colombia). \\ Correo-e: gcastano@funlam.edu.co
}

\section{Resumen}

Se estudia la relación entre consumo de drogas y prácticas sexuales en adolecentes de la ciudad de Medellín (Colombia). Mediante muestreo probabilístico aleatorio y por conglomerados, se obtuvo una muestra representativa de 955 adolescentes entre los 14 y 17 años de edad pertenecientes a colegios públicos y privados de la ciudad de Medellín de los grados $9^{\circ}, 10^{\circ}$ y $11^{\circ}$. Los resultados muestran que la prevalencia general de relaciones sexuales bajo el efecto de alcohol o drogas es del 43,67\%. Las drogas más usadas para tener prácticas sexuales son el alcohol, la marihuana, el popper, la cocaina y el éxtasis. Consumir alcohol o drogas y tener prácticas sexuales presenta una asociación estadistica significativa ( $p=$ .001). Las practicas sexuales mas frecuentes bajo el efecto de sustancias psicoactivas son las exploratorias (manoseo, caricias) 71\%; la penetrativa vaginal (63.67\%), el sexo oral (45.30\%) y la masturbación (19.59\%). Con respecto a tener prácticas sexuales bajo efecto de alcohol o drogas y usar métodos de protección, el 55,9\% de los jóvenes usan siempre el condón, el $37,3 \%$ lo usan algunas veces y el 6,8\% nunca lo usan. Este estudio prueba lo ya constatado por otras investigaciones donde se muestra la alta asociación estadistica que hay entre el consumo de drogas y las prácticas sexuales, pero da cuenta que no existe asociación estadistica significativa entre tener practicas sexuales bajo efectos de alcohol o drogas y el uso o no uso de métodos de protección, lo que constituye su hallazgo mas importante.

Palabras clave: prácticas sexuales, consumo de drogas, adolescentes, Colombia.

\section{Abstract}

The purpose of this research project was to study the relationship between drug consumption and sexual practices in teenagers in the city of Medellin, Colombia. A transversal studied was designed in order to identify the variables related to having had sexual intercourse under the effects of drugs or alcohol. The sample was made up of 955 teenagers between 14 and 17, who were in 9th, 10th and 11th grades in public and private schools in the city of Medellin. The results show that the prevalence of drug and alcohol influence in sexual intercourse is 43,67\%. The most common drugs used for sexual practices are alcohol, marihuana, popper, cocaine and ecstasy. Consuming alcohol or drugs and having sexual practices shows an associated meaningful statistic $(p=.001)$. The more common sexual practices under psychoactive substances are the exploratory ones (caresses and touching) (71\%), vaginal penetration (63.67\%), oral sex (45.30\%) and masturbation (19.59\%). Regarding the protection methods during sexual intercourse under drug or alcohol influence 55,9\% always use a condom, 37,3\% sometimes use it, and 6,8\% never do it. This study proves what had been previously established by other research projects that show a high statistic association between drug consumption and sexual practices, but realizes that there is no statistically significant association between sexual practices under the influence of alcohol or drugs and the use or non-use of protective methods, which is the most important finding

Key words: sexual practices, drug consumption, teenagers. 
a sexualidad, el consumo de alcohol y drogas en los adolescentes figuran desde hace tiempo como una prioridad para la salud pública. La preocupación radica en reducir los riesgos de infecciones de transmisión sexual (ITS), el virus de la inmunodeficiencia humana y los embarazos tempranos no deseados. (Juarez y Gayet, 2005). Debido a que la adolescencia es una etapa de cambios en la cual el sujeto se encuentra en constante experimentación consigo mismo y con lo que lo rodea, las probabilidades de entrar en terreno del consumo de drogas y de ejercer su sexualidad en forma riesgosa aumentan.

Según Giddens (1995) los valores ante la sexualidad han cambiado en las últimas décadas en algunos entornos socioculturales y esto parece estar pasando en el nuestro. Los jóvenes colombianos actuales están siendo educados a partir de valores más liberales que han dado paso a otro sistema regulador de la sexualidad, basado en la responsabilidad y la autonomía. La importancia que dan los jóvenes a la sexualidad ha aumentado, según el argumento de Elzo (2006), de forma paralela a como aumentan los deseos de objetivos vinculados a un placer inmediato y sin compromisos, en donde también muchas veces están incluidas las drogas.

En la literatura científica hay evidencia empírica que apoya la idea de una relación significativa entre el consumo de sustancias psicoactivas y conductas sexuales de alto riesgo durante la adolescencia y la adultez (Breen et al., 2006; Brook, 2002; Calafat, Fernández y Juan, 2001; Elliot et al., 1998 y Ford y Norris, 1994); sin embargo, otros autores como Liau et al. (2002) dudan de esta asociación argumentando que muchos estudios basan la validez de sus resultados, teniendo en cuenta sólo los autorreportes de los adolescentes con respecto al uso de drogas y las conductas sexuales de alto riesgo, lo que podría sesgar o por lo menos generar algún tipo de error estadístico en interpretación de los resultados que se obtienen de las muestras estudiadas.

Los efectos de las diferentes drogas sobre el deseo y el acto sexual ha sido estudiado por diversos autores: el alcohol por Bellis y Hughes (2004), el cannabis por Poulin y Graham (2001), la cocaína por Pechansky et al. (2000); el éxtasis por Capdevila (1995); las anfetaminas por Souza y Oliveira (1998); los poppers por Castro (2003) o el GHB por Fernández y Hernández (2003). Muchas de estas drogas se valoran y consumen por sus efectos sobre las prácticas sexuales (Calafat, Juan, Becoña y Mantecón, 2008) y su consumo se asocia a menudo con conductas sexuales de riesgo (Bellis y Hughes, 2004) y experiencias sexuales pasajeras (Lomba, 2006).

Sin embargo, hay bastante incertidumbre empírica sobre la dinámica concreta de la relación de estas drogas con las conductas sexuales de riesgo pues intervienen muchas variables. Por ello algunos autores consideran que la investigación debería centrarse en la relación entre la "interacción farmacológica de las drogas, las conductas de riesgo sexual y factores sociales y culturales más amplios que influyen en la actividad sexual y en el consumo de drogas en diferentes contextos sociales" (Rhodes y Stimson, 1994).
Sobre los riesgos asociados a las practicas sexuales y el efecto de sustancias psicoactivas, el Instituto Nacional de Abuso de Alcohol y Alcoholismo de los Estados Unidos de América reporta que las personas con desórdenes de consumo de alcohol tienen mayor probabilidad de contraer infección por VIH y otras enfermedades de transmisión sexual que la población general (NIAAA, sf), debido a relaciones sexuales sin protección, múltiples parejas, relaciones con trabajadoras sexuales y sexo a cambio de alcohol o drogas (De Oliveira, Pereira y Reis, 2001).

Este trabajo pretende dar cuenta de las prácticas sexuales de los adolescentes bajo efecto de drogas y revisar la hipótesis, ya confirmada por otros estudios en los que se afirma que los consumidores de sustancias psicoactivas tienen mayor probabilidad de realizar practicas sexuales de riesgo, sobre todo por el no uso de métodos de protección. Como aporte del estudio a la evidencia que ya se tiene sobre esta relación, se destacan los motivos que los adolescentes aducen para tener relaciones sexuales bajo el efecto de alcohol o drogas, el tipo de prácticas que realizan y un hallazgo que va en contravía de lo hasta ahora establecido.

\section{Método}

Se diseñó un estudio correlacional descriptivo y analítico, de tipo transversal

\section{Muestra}

Adolescentes de ambos sexos matriculados en los grados $9^{\circ}, 10^{\circ}$ y $11^{\circ}$ de secundaria, entre los 14 y los 17 años en los colegios públicos y privados de la ciudad de Medellin, registrados en la Secretaria Municipal de Educación en el año 2010. Solo se incluyeron colegios del área urbana. Acordes con la legislación colombiana vigente para investigación en salud, el estudio representaba un mínimo riesgo para los participantes (Minsalud, 1993). De acuerdo con la Declaración de Helsinki, los estudiantes participaron en forma voluntaria y se les garantizó el anonimato a las respuestas. La muestra se estableció mediante método probabilístico aleatorio y por conglomerados. Se tomó como universo poblacional el número total de estudiantes matriculados de $8^{\circ}, 9^{\circ}, 10^{\circ}$ y $11^{\circ}$ en el año 2010 de colegios públicos y privados de la ciudad de Medellín con edades comprendidas entre los 14 y 17 años; el total del universo poblacional fue de 91.857 estudiantes. El tamaño muestral incluido fue de 955 estudiantes usando un intervalo de confianza del 95\%.

\section{Instrumentos}

Los estudiantes diligenciaron en el aula de clase un formulario construido por los investigadores a partir de la revisión de la literatura, el cual fue auto -administrado. Este incluía puntos que abordaban los aspectos socio - demográficos, tipo de colegio, antecedentes familiares, actitudes cognitivas y emocionales en relación con el consumo de alcohol y drogas y prácticas sexuales, variables relacionadas con los 
grupos, consumo de drogas, prácticas sexuales, métodos de protección usados, razones para tener prácticas sexuales bajo el efecto de alcohol o drogas.

\section{Análisis de datos}

Los datos se analizaron en el paquete estadístico SPSS 18.0. Se realizaron pruebas de significancia estadísticas con el Chi Cuadrado de Mantel y Haenzel o test de tendencias.

\section{Resultados}

En el estudio participaron 955 estudiantes que estaban matriculados en instituciones educativas públicas y privadas de la ciudad de Medellín, en el año 2011, 54,8\% hombres y el $45,2 \%$ mujeres, con edades comprendidas entre los 14 y los 18 años, la mayoría ubicados entre los 15 y los 16 años, donde se encontraba $62,7 \%$ de la población. El mayor peso porcentual de estudiantes se encontró en los estratos socioeconómicos ${ }^{1}$ bajos, 1,2 y $3(69,43 \%)$, y en los colegios públicos $(68,9 \%)$, matriculados en los grados $9^{\circ}(33.3 \%), 10^{\circ}$ $(29,3 \%)$ y $11^{\circ}(30,4 \%)$, respectivamente (ver Tabla 1$)$.

Tabla 1. Características sociodemográficas de la población sujeto de estudio $(\mathrm{N}=955)$

\begin{tabular}{ccc} 
VARIABLE & FRECUENCIA & PORCENTAJE \\
\hline GENERO & 523 & \\
Hombre & 432 & $54,8 \%$ \\
Mujer & & $45,2 \%$ \\
& & \\
\hline EDAD & 210 & $22,0 \%$ \\
14 & 272 & $28,5 \%$ \\
15 & 327 & $34,2 \%$ \\
16 & 135 & $14,1 \%$ \\
17 & 7 & $0,7 \%$ \\
18 & Sin dato (4) & Sin dato $(0,4 \%)$
\end{tabular}

\begin{tabular}{ccc}
\hline ESTRATO SOCIOECONOMICO & & \\
$1-2--3$ & 663 & $69.42 \%$ \\
$4-5-6$ & 273 & $28,58 \%$ \\
& Sin dato ( 19) & $2,0 \%$ \\
& & \\
\hline TIPO DE INSTITUCION EDUCATIVA & & \\
PÚBLICA & 658 & $68,9 \%$ \\
PRIVADA & 287 & $30,1 \%$ \\
& Sin dato (10) & $1,0 \%$ \\
\hline & & \\
\hline GRADO OUE CURSAN & & \\
$8^{\circ}$ & 59 & $6,2 \%$ \\
$9^{\circ}$ & 318 & $33,3 \%$ \\
$10^{\circ}$ & 280 & $29,3 \%$ \\
$11^{\circ}$ & 290 & $30,4 \%$ \\
& Sin Dato (8) & $0,8 \%$ \\
\hline
\end{tabular}

1 La estratificación socioeconómica colombiana, es una clasificación de los domicilios o viviendas a partir de sus caracteristicas físicas y de su entorno, en seis grupos o estratos, correspondiendo los grupos 1, 2 a las clases bajas, 3, 4 a la media y 5, 6 a las altas. Fuente: Dirección Administrativo Nacional de Estadística DANE
Del total de adolescentes encuestados (955), 561 $(58,7 \%)$ manifiestan haber tenido prácticas sexuales. De éstos el $43,67 \%$ (245) las ha tenido bajo efectos de alcohol o drogas, lo que equivale a plantear que de cada cuatro estudiantes, uno ha tenido las prácticas en estas condiciones

Entre los que han tenido prácticas sexuales $(N=561)$ la edad de inicio que aparece con mayor frecuencia son los 14 años $(15,3 \%)$, seguida por los jóvenes de $15(12,8 \%)$ y 13 $(9,6 \%)$ años de edad. Es interesante anotar que se identificó un porcentaje de relaciones sexuales iniciadas antes de los 10 años, representada en un 2\%, que si bien para la muestra puede ser bajo, por su condición, es importante resaltarlo en tanto los niños y niñas en estas edades están en un proceso de desarrollo biológico y psicológico.

Las drogas más frecuentemente utilizadas por los adolescentes para tener relaciones sexuales son el alcohol en el 79,8\% de los casos, seguidas por la marihuana 31.83\%, cocaina $7.75 \%$, poppers $8.97 \%$, Extasis $5.71 \%$, benzodiacepinas y cacao sabanero $4.08 \%$, LSD $3.67 \%$, hongos 3,26\%, inhalantes (bóxer, sacol) $2.85 \%$ y heroína $2.04 \%$. Con cifras por debajo del $2 \%$ y por encima de 1\%, aparecen GHB, Ketamina, 2CB y Metanfetamina (Cristal). Lo cual evidencia la diversidad de drogas al alcance de la población sujeto de estudio, que se convierte en un dato relevante en tanto son menores de edad de acuerdo con la legislación colombiana, es decir, menores de 18 años.

Consumir alcohol o drogas y tener relaciones sexuales presentan una asociación estadísticamente significativa, ( $p$ $<.001)$. El 62.4\% de los adolescentes que han consumido una sustancia psicoactiva ha tenido estas practicas, frente a un $29,4 \%$ que habiendo consumido drogas, no lo han hecho.

Los motivos que aducen los 245 estudiantes, para tener prácticas sexuales bajo el efecto de alcohol o drogas, pueden verse en la tabla 2. El alcohol que es la sustancia más consumida, es utilizado, por gusto en el $51.42 \%$ de los casos, seguido de facilitar el inicio en la práctica sexual 47.34\%, aumentar la excitación 25.71\%; prolongar la relación sexual $19.59 \%$ y evitar la eyaculación precoz en el $12.65 \%$ de los casos. La marihuana, sustancia que le sigue en frecuencia, como la preferida para tener prácticas sexuales, es consumida por gusto $15.10 \%$, para aumentar la excitación $13.46 \%$, prolongar la relación sexual $7.34 \%$, facilitar el inicio $6.53 \%$ y evitar la eyaculación precoz en el $6.12 \%$ de los casos. La cocaína es utilizada con mayor frecuencia para prolongar la relación $4.08 \%$ y por gusto $3.67 \%$; el Popper es consumido para aumentar la excitación 2.44\%; las benzodiacepinas por gusto 2.04\%; el éxtasis para aumentar la excitación y por gusto $1.63 \%$ y la heroína para evitar la eyaculación precoz y prolongar la relación en un $0.40 \%$, de los casos.

Entre los estudiantes que ya tienen actividad sexual, las prácticas más frecuentes estando bajo el efecto del alcohol o las drogas, son las exploratorias (71.02\%), la penetrativa vaginal (63.67\%), el sexo oral (45.30\%) y la masturbación $19.59 \%$. Con una menor frecuencia también practican el sexo anal $12.24 \%$, sexo con juguetes eróticos $8.16 \%$, sexo en tríos $6.96 \%$, orgias $4.89 \%$ y cibersexo $4.08 \%$. 
Tabla 2. MOTIVOS PARA TENER RELACIONES SEXUALES BAJO EL EFECTO DE ALCOHOL O DROGAS. $(\mathrm{N}=245)$

\begin{tabular}{|c|c|c|c|c|c|c|c|c|c|c|c|c|c|c|}
\hline \multirow[t]{2}{*}{ MOTIVOS } & \multicolumn{2}{|c|}{$\mathrm{ALCOHOL}$} & \multicolumn{2}{|c|}{$\mathrm{THC}$} & \multicolumn{2}{|c|}{ COCAINA } & \multicolumn{2}{|c|}{ EXTASIS } & \multicolumn{2}{|c|}{ POPPER } & \multicolumn{2}{|c|}{ BZD } & \multicolumn{2}{|c|}{ HEROÍNA } \\
\hline & $\mathrm{F}$ & $\%$ & $\mathrm{~F}$ & $\%$ & $\mathrm{~F}$ & $\%$ & $\mathrm{~F}$ & $\%$ & $\mathrm{~F}$ & $\%$ & $\mathrm{~F}$ & $\%$ & $\mathrm{~F}$ & $\%$ \\
\hline Facilitar el inicio & 116 & $47,34 \%$ & 16 & $6,53 \%$ & 5 & $2,04 \%$ & 1 & $0,40 \%$ & 3 & $1,22 \%$ & 1 & $0,40 \%$ & 0 & - \\
\hline Aumentar excitación & 63 & $25,71 \%$ & 33 & $13,46 \%$ & 6 & $2,44 \%$ & 4 & $1,63 \%$ & 6 & $2,44 \%$ & 4 & $1,63 \%$ & 0 & - \\
\hline Prolongar relación & 48 & $19,59 \%$ & 18 & $7,34 \%$ & 10 & $4,08 \%$ & 1 & $0,40 \%$ & 2 & $0,81 \%$ & 2 & $0,81 \%$ & 1 & $0,40 \%$ \\
\hline Evitar eyaculación precoz & 31 & $12,65 \%$ & 15 & $6,12 \%$ & 6 & $2,44 \%$ & 0 & - & 2 & $0,81 \%$ & 1 & $0,40 \%$ & 1 & $0,40 \%$ \\
\hline Por gusto & 126 & $51,42 \%$ & 37 & $15,10 \%$ & 9 & $3,67 \%$ & 4 & $1,63 \%$ & 2 & $0,81 \%$ & 5 & $2,04 \%$ & 1 & $0,40 \%$ \\
\hline
\end{tabular}

Tabla 3. MÉTODOS ANTICONCEPTIVOS USADOS POR LOS JÓVENES $(\mathrm{N}=470)$

\begin{tabular}{l|c|c|c}
\hline \multirow{2}{*}{ Método } & \multicolumn{3}{|c}{ Respuestas } \\
\cline { 2 - 4 } Anticonceptivo & Siempre & Algunas veces & Nunca \\
\hline Condón & 263 & 168 & 39 \\
& $27.5 \%$ & $17.6 \%$ & $4.1 \%$ \\
Píldora o anticoncep- & 60 & 79 & 331 \\
tivos orales & $6.3 \%$ & $8.3 \%$ & $34.7 \%$ \\
Marcha atrás & 77 & 102 & 291 \\
& $8.1 \%$ & $10.7 \%$ & $30.5 \%$ \\
Inyección & 41 & 39 & 390 \\
& $4.3 \%$ & $4.1 \%$ & $40.8 \%$ \\
Cremas/geles & 21 & 31 & 418 \\
Pildora del día después & $2.2 \%$ & $3.2 \%$ & $43.8 \%$ \\
& 56 & 47 & 365 \\
Dispositivo & $6.1 \%$ & $4.9 \%$ & $38.2 \%$ \\
& 34 & 36 & 400 \\
Otro & $3.6 \%$ & $3.8 \%$ & $41.9 \%$ \\
& 26 & 9 & 435 \\
& $2.7 \%$ & $0.9 \%$ & $45.5 \%$ \\
\hline
\end{tabular}

El $83,77 \%$ de los adolescentes, que han tenido prácticas sexuales con penetración, han usado métodos de protección o planificación. De estos el $60,8 \%$ lo usan siempre y el $53,5 \%$ algunas veces. El condón es el método anticonceptivo más utilizado por los jóvenes, siempre $(27,5 \%)$ y algunas veces $(17,6 \%)$; seguido del método de la marcha atrás (eyacular por fuera), siempre $(8,1 \%)$ y algunas veces $(10,7 \%)$ y la píldora o anticonceptivos orales, siempre $(6,3 \%)$ y algunas veces $(8,3 \%)$. Llama la atención el uso de la pastilla del día después, siempre $(6,1 \%)$ y algunas veces $(4,9 \%)$. (Tabla 3$)$.

Las razones mas relevantes que los adolescentes argumentan para no usar métodos anticonceptivos o de protección son: el tener una práctica sexual inesperada y no tener el condón a la mano $(16,92 \%)$; tener una pareja estable y de mucha confianza $(13,89 \%)$, se le olvida utilizarlos $(5,52 \%)$, falta de información para usarlos (2,13\%). Llama la atención que ningún adolescente considero que estar bajo efecto de alcohol o drogas es una razón para no protegerse (Tabla 4).

Las consecuencias reportadas por los jóvenes que han tenido prácticas sexuales y que no han usado métodos de protección o planificación, fueron de 2,8 \% para embarazo y 2,0\% infecciones de transmisión sexual.
Con respecto a tener prácticas sexuales bajo efecto de alcohol o drogas y usar de métodos de protección, el 55,9\% de los jóvenes usan siempre el condón, el 37,3\% lo usan algunas veces y el 6,8\% nunca lo usan. No se encontró asociación estadísticamente significativa, $(p=.711)$ entre tener relaciones sexuales bajo el efecto de alcohol o drogas o estar sobrios y el uso de métodos de protección. (Tabla 5).

\section{Discusión}

En Colombia, la mayoría de los estudios disponibles, incluidos éste, muestran que la prevalencia de relaciones sexuales antes de los 18 años es alta, siendo mayor en los hombres. (Ceballos y Campo-Arias, 2005; Vinaccia et al., 2007; Tuesta et al., 2007; Barrios et al., 2007). El promedio de edad de inicio en la actividad sexual en estos trabajos son los 14 años, lo cual coincide con lo hallado por nosotros.

De otro lado y a diferencia de lo encontrado por Ceballos y Campo-Arias (2007), en adolescentes de Santa Marta (Colombia) donde hallo una mayor frecuencia de prácticas sexuales en los estudiantes de colegios privados, de mayor edad y mayor escolaridad, en nuestro trabajo las mayores prácticas sexuales se presentaron en los colegios públicos, en edades mas tempranas y en los grados intermedios ( $9^{\circ}$ y $10^{\circ}$ ).

En nuestro estudio las prácticas sexuales bajo el efecto de alcohol o drogas alcanzan el $43.67 \%$, cifra un poco más baja a la reportada por Parra-Villarroel y Pérez-Villegas (2010) en Chile, quienes encontraron que el $48 \%$ de los adolescentes que beben alcohol han tenido relaciones sexuales versus un $14.1 \%$ de los que no ingieren esta sustancia, lo mismo que ocurre en cuanto al consumo de otras sustancias.

En todos los estudios, incluido el nuestro, el alcohol es la sustancia más consumida y que más influye en la conducta sexual. (Abrahamson, 2004; Martin, 2001; Lameiras et al., 2005; Gaspar, Gaspar de Matos et al., 2006; Reis y Matos, 2008; Da Silva, Rumbao, Benitez, García y Rodríguez, 2001). El alcohol es usado para reforzar la confianza (Abrahamson, 2004) y rebajar las inhibiciones (Martin, 2001). En este trabajo se hallo que lo hacen buscando estos mismos beneficios y para aumentar la excitación. Con la marihuana también se busca facilitar el inicio, deshinbirse y aumentar la excitación y fue la segunda droga preferida por los adolescentes de esta investigación, diferente a lo encontrado por Calafat, Juan, 
Tabla 4. RAZONES PARA EL USO Y NO USO DE MÉTODOS ANTICONCEPTIVOS O DE PROTECCIÓN EN LOS ADOLESCENTES. (N=561).

\begin{tabular}{|c|c|c|c|c|}
\hline \multirow[t]{2}{*}{ Razones } & \multicolumn{2}{|c|}{$\begin{array}{l}\text { SI, ES UNA RAZON PARA NO HABERLO } \\
\text { USADO }\end{array}$} & \multicolumn{2}{|c|}{$\begin{array}{l}\text { NO, ES UNA RAZON PARA NO HABERLO } \\
\text { USADO }\end{array}$} \\
\hline & Frecuencia. & $\%$ & Frecuencia. & $\%$ \\
\hline $\begin{array}{l}\text { Ha tenido relaciones con una pareja estable y de con- } \\
\text { fianza }\end{array}$ & 78 & $13,89 \%$ & 170 & $30,29 \%$ \\
\hline Me lo prohíben mis creencias & 3 & $0,5 \%$ & 121 & $21,56 \%$ \\
\hline $\begin{array}{l}\text { Todos me caen, me sientan mal y me da miedo los efecto } \\
\text { secundarios }\end{array}$ & 11 & $1,95 \%$ & 259 & $46,16 \%$ \\
\hline Deseo tener un hijo & 2 & $0,35 \%$ & 122 & $21,74 \%$ \\
\hline Me falta información para usarlos & 12 & $2,13 \%$ & 134 & $23,88 \%$ \\
\hline Me resulta muy difícil conseguirlos & 7 & $1,24 \%$ & 139 & $24,77 \%$ \\
\hline Mi pareja no quiere usarlos & 8 & $1,42 \%$ & 138 & $24,59 \%$ \\
\hline Se me olvida utilizarlos & 31 & $5,52 \%$ & 115 & $20,49 \%$ \\
\hline Estaba demasiado borracho o drogado & - & $0 \%$ & 146 & $26,02 \%$ \\
\hline La relación fue inesperada y no los tenia a mano & 95 & $16,92 \%$ & 197 & $35,1 \%$ \\
\hline
\end{tabular}

Tabla 5. Tener prácticas sexuales bajo el efecto del alcohol o la droga y uso de condón

\begin{tabular}{|c|c|c|c|c|c|c|}
\hline \multicolumn{2}{|c|}{$\begin{array}{l}\text { Tener practicas sexuales bajo efecto de alcohol o drogas y } \\
\text { uso de métodos de protección }\end{array}$} & \multicolumn{3}{|c|}{ Uso de condón } & \multirow[t]{2}{*}{ Total } & \multirow[t]{2}{*}{$x^{2}$} \\
\hline \multirow{5}{*}{$\begin{array}{l}\text { Ha tenido prácticas sexuales bajo el efecto } \\
\text { del alcohol o la droga: }\end{array}$} & \multirow{3}{*}{ Si } & Siempre & Algunas veces & Nunca & & \\
\hline & & 123 & 82 & 15 & 220 & \multirow[t]{4}{*}{0,711} \\
\hline & & $55,9 \%$ & $37,3 \%$ & $6,8 \%$ & $100,0 \%$ & \\
\hline & \multirow[t]{2}{*}{ No } & 113 & 66 & 16 & \multirow[b]{2}{*}{$100,0 \%$} & \\
\hline & & $57,9 \%$ & $33,8 \%$ & $8,2 \%$ & & \\
\hline \multicolumn{2}{|l|}{ Total } & 236 & 148 & 31 & \multicolumn{2}{|l|}{415} \\
\hline
\end{tabular}

Becoña, Mantecón (2008), quienes hallaron controversias con respeto al uso de marihuana en las prácticas sexuales. En nuestro estudio, la cocaína ocupo el tercer lugar, como sustancia preferida para tener relaciones sexuales por debajo inclusive del Popper, que es usado para aumentar la excitación y por gusto, igual a lo descrito por Castro (2003). Los consumidores de heroína lo que buscan es evitar la eyaculación precoz, igual a lo hallado por Castaño y Calderón (2010).

El éxtasis, que fue mitificada en las décadas del 80 y 90 del siglo pasado como la droga del amor, fue muy poco utilizado por los adolescentes de este estudio, muy similar a lo encontrado por Calafat, Juan, Becoña y Mantecón (2008) y distinto a lo referido por Zemishlany et al. (2001) y Sumnall et al. (2006), quienes hallaron un uso importante de esta sustancia en las prácticas sexuales.

Aunque el condón es el método de protección más utilizado en nuestro estudio, solo lo usan, el $27,5 \%$, siempre, el $17,6 \%$ algunas veces y el 4,1\% nunca. Lo cual tiene relación con algunas consecuencias negativas que refieren los adolescentes, por realizar prácticas sexuales sin protección, tales como el embarazo $(2,8 \%)$ y las ETS $(2,0 \%)$, cifras muy similares a las reportadas por Agudelo (2005). El uso consistente de preservativos se presenta como la única forma de prevenir el riesgo de esta enfermedades (Rodríguez, García, Cabrera, Romo, García y Gutiérrez, 2006).

Otros autores como Lomba, Apóstalo y Mendes (2009) han encontrado que el 46,7\% de su muestra, consideraba a las drogas y el alcohol como factores claves en las practicas de sexo no seguro. En nuestro caso ningún adolescente, considero que estar bajo efecto de alcohol o drogas era una razón para no protegerse en las relaciones sexuales. Este resultado coincide con lo descrito por Antón-Ruiz y Espada (2009) quienes tampoco hallaron diferencias significativas con respecto al uso del condón, bajo el efecto de alcohol y cannabis o cuando se esta sobrio. Este es un aspecto que seguramente necesita investigarse más, pues es muy relevante respecto a las medidas preventivas que se deben promover, máxime cuando es tan frecuente la asociación de sexualidad y consumo de sustancias.

Hay que anotar que, una limitación de éste trabajo lo constituye el ser un estudio transversal y que se haya limitado el uso a pruebas estadísticas clásicas, como la mayoría de los estudios existentes. 
Reconocimientos: Agradecemos a la Secretaria de Educación del Municipio de Medellín el habernos ayudado en los contactos con los colegios para recoger la información, así como a los docentes, directores de las instituciones participantes y a los jóvenes que diligenciaron voluntariamente la encuesta; a Marcela Montoya por la ayuda en el procesamiento de los datos; a los estudiantes del programa de Psicologia y de la Especialización en Farmacodependencia de la Fundación Universitaria Luis Amigó, por habernos ayudado en la recogida de la información.

Ha sido financiado por el Centro de investigaciones de la Fundación Universitaria Luis Amigó, convocatoria interna I - 2010.

\section{Conflicto de intereses}

Ninguno.

\section{Referencias}

Abrahamson, M. (2004). Alcohol in courtship contexts: focus-group interviews with young Swedish women and men. Contemporay Drug Problems, 31, 3-29.

Agudelo, L. (2005). Redescubrir: una mirada a la salud de las y los jóvenes: Prevalencia de embarazo adolescente, sintomas sugestivos de ITS, consumo y adicción a sustancias psicoactivas, comportamientos violentos y factores relacionados en mujeres de 14 a 19 años de Medellín. Secretaria de Salud, Alcaldía de Medellín.

Antón-Ruiz, F. A. y Espada, J. P. (2009). Consumo de sustancias y conductas sexuales de riesgo para la transmisión del VIH en una muestra de estudiantes universitarios. Anales de psicología, 25, 344-350.

Barrios, Y. I., Berrío, V.C. y Gómez, E. M. (2007). Relaciones sexuales en adolescentes de una institución de Cartagena, Colombia. Archivos de Salud, 1, 39-45.

Bellis, M. y Hughes, K. (2004). Pociones sexuales. Relación entre alcohol, drogas y sexo. Adicciones, 16, 249-57.

Breen, C., Degenhart, L., Kinner, S., Bruno, R., Jenkinson, R., Matthews, A. y Newman, J. (2006) Alcohol use and risk taking among regular ecstasy users. Substance Use and Misuse, 4, 1095-1109.

Brook, D.W., Brook, J.S., Pahl, T. y Montoya, I. (2002).The longitudinal relationship between drug use and risky sexual behaviors among Colombian adolescents. Archives of Pediatric Adolescence Medicine, 156, 1101-1107.

Calafat, A., Fernández, C., y Juan, M. (2001). Uso recreativo de cocaína y asunción de riesgos: resultados convergentes de cuatro muestras. Adicciones, 13 (Supl. 2), 123-146.

Calafat, A., Juan, M., Becoña, E. y Mantecón, A. (2008). Qué drogas se prefieren para las relaciones sexuales en contextos recreativos. Adicciones, 20, 37-48.
Capdevila, M. (1995). MDMA o el éxtasis químico. Barcelona: Los Libros de la Liebre de Marzo.

Castaño, G.A. y Calderon, G.A. (2010). Comorbilidad asociada en un grupo de consumidores de heroína de Medellín. Revista Colombiana de Psiquiatría, 39, (Sup/1), 93-111.

Castro, D. (2003). Nuevas infecciones por el VIH: perfil del seroconvertor reciente (2000-2002). Publicación Oficial de la Sociedad Española Interdisciplinaria del SIDA, 14.

Ceballos, G.A. y Campo-Arias, A. (2007). Relaciones sexuales en adolescentes escolarizados de Santa Marta, Colombia: una encuesta transversal. Colombia Médica, 38, 185-90.

Ceballos, G.A. y Campo-Arias, A. (2005). Relaciones sexuales en estudiantes adolescentes de Santa Marta Colombia. Factores asociados. Duazary, 2, 4-10.

Cortés, D., Gallego, J., y Maldonado, D. (2011). On the design of education conditional cash transfer programs and non education outcomes: the case of teenage pregnancy. Universidad del Rosario. Facultad de Economía. Documentos de trabajo, 10.

Da Silva, M.I., Rumbao, F., Benitez, G., García, R. y Rodríguez, T. (2001). Consumo de alcohol y relaciones sexuales en adolescentes del sexo femenino. Revista de La Facultad de Medicina, 24, 135-139.

De Oliveira, L.C., Pereira, R.G. y Reis, U.C. (2001). Prevalence of human immune deficiency virus infection in alcoholics. Memorias do Instituto Oswaldo Cruz, 96, 21-23.

Elliott, L., Morrison, A., Ditton, J., Farrall, S., Short, E., Cowan, L. y Gruer, L. (1998). Alcohol, drug use and sexual behaviour of young adults on a Mediterranean dance holliday. Addiction Research, 6, 319-340.

Elzo, J. (2006). Valors i identitats dels joves. Una aproximación empírica. Revista del Centre D'Estudis Jordi Pujol, 9. Recuperado Setiembre 15 de 2010. Disponible en: http://www.jordipujol.cat/ files/articles/Javier_Elzo.pdf.

Fernández, P. y Hernández, I. (2003). Características farmacológicas de las drogas recreativas (MDMA y otras anfetaminas, ketamina, GHB, LSD y otros alucinógenos). Adicciones, 15, 51-75.

Ford, K. y Morris, A. (1994). Urban minority youth: Alcohol and marihuana use and exposure to unprotected intercourse. Journal Acquired Immune Deficiency Syndrome, 7, 389-396.

Gaspar, T., Gaspar de Matos, M., Gonçalves, A., Ferreira, M. y Linares, F. (2006). Comportamentos sexuais, conhecimentos e atitudes face Ao VIH/SIDA em adolescentes migrantes. Psicologia, Saúde Et Doenças, 7, 299-316.

Giddens, A. (1995). La transformación de la intimidad. Sexualidad, amor y erotismo en las sociedades modernas. Madrid: Gráficas Rógar.

Juárez, F., y Gayet, C. (2005). Salud Sexual Reproductiva de los adolescentes en México: Un nuevo marco de análisis para la evaluación y diseño de políticas. Papeles de población, 45, 177219

Lameíras-Fernández, M., Núñez-Rodríguez, A., Rodríguez - Castro, Y., Bretón- López, J. y Agudelo, D. (2005). Actividad sexual y uso 
del preservativo masculino en jóvenes universitarios gallegos. Clínica y salud, 16, 253-267.

Liau, A., DiClemente, R.J., Wingood, G.M., Crosby, R.A., Williams, K.M., Harrington, K, et al. (2002). Association between biologically confirmed marijuana use and laboratory confirmed sexually transmitted diseases among African-American adolescents females. Sexually Transmitted Diseases, 29, 387390.

Lomba, L. (2006). 0 consumo recreativo de ecstasy In: Relvas, J., Lomba, L. y Mendes, F. Novas drogas e ambientes recreativos. Loures: Lusociência, 45-60.

Lomba, L., Apostolo, J. y Mendes, F. (2009). Consumo de drogas, alcohol y conductas sexuales en los ambientes recreativos nocturnos de Portugal. Adicciones, 21, 309-325.

Martin, L. (2001). Alcohol, sex and gender in late medieval and early modern Europe. New York: Palgrave Macmillan.

Minsalud (1993). Resolución 008430 de 1993, por la cual se establecen las normas cientificas, técnicas y administrativas para la investigación en salud. Santafé de Bogotá: Ministerio de Salud.

NIAAA (sf). Alcohol and HIV/AIDS. Recuperado el 10 de agosto de 2011. Disponible en : http://pubs.niaaa.nih.gov/publications/ EABReport/EABReport.htm

Parra-Villarroel., J. y Pérez-Villegas., R. (2010). Comportamiento sexual en adolescentes y su relación con variables biosociales. Perinatología y Reproducción Humana, 24, 7-19

Pechansky, F., Inciardi, A., Surratt, H., Lima, S., Kessler, P., Soibelman, M., et al. (2000). Estudo sobre as características de usuários de drogas injetáveis que buscamatendimentoem Porto Alegre. Revista Brasileira de Psiquiatria, 22, 164-71.

Poulin, C. y Graham, L. (2001). The association between substance use,unplanned sexual intercourse and other sexual behaviours among adolescent students. Addiction, 96, 607-21.

Reis, M. y Matos, M. (2008). Contracepção em jovens universitarios portugueses. Análise Psicológica, 1, 71-79.

Rhodes, T. y Stimson, G. (1994). What is the relationship between drug taking and sexual risk? Social relations and social research. Sociology of Health \& IIIness, 16, 209-228.

Rodríguez, A., García, M.H., Cabrera, A., Romo, N., García, J.M. y Gutiérrez, J.L. (2006) Tienen adolescentes y jóvenes que consumen drogas no inyectadas mayor probabilidad de transmisión sexual del VIH? Adicciones, 18, 61-72.

Souza, D. y Oliveira, D. (1998). 0 perfil epidemiológico do uso de drogas entre estudantes de $1^{\circ}$ e $2^{\circ}$ graus da rede estadual de ensino de Cuiabá, Brasil. Saúde Pública, 14, 391-400.

Sumnall, H.R., Cole, J.C. y Jerome, L. (2006). The varieties of ecstatic experience: an exploration of the subjective experiences of ecstasy. Journal of Psychopharmacology, 20, 670-682.

Tuesta, R., Navarro, E., Medina, A., Molina, I., Náder, N. y Num, L. (2007). Hábitos de salud de los escolares de una institución pública en el municipio de Soledad (Atlántico). Salud Uninorte, $23,135-4$.
Vinaccia, S., Quiceno, J.M., Gaviria, A.M., Soto, A.M., Gil, M.D. y Ballester, R. (2007). Conductas sexuales de riesgo para la infección por $\mathrm{VIH} /$ sida en adolescentes colombiano. Terapia Psicológica, 25, 39-50.

Zemishlany, Z., Aizenberg, D. y Weizman, A. (2001). Subjective effects of MDMA ('Ecstasy') on human sexual function. European Psychiatry, 16, 127-130. 
